in vivo $35: 3581-3584(2021)$

doi:10.21873/invivo.12662

\title{
Sella Turcica Shape in Fragile X Syndrome
}

\author{
REINHARD E. FRIEDRICH \\ Department of Oral and Craniomaxillofacial Surgery, \\ Eppendorf University Hospital, University of Hamburg, Hamburg, Germany
}

\begin{abstract}
Background: Fragile X syndrome (FXS) is a relatively common syndrome with numerous, multifaceted, and often unremarkable findings. Psychic alterations are at the forefront of treatment needs. Previous studies have provided evidence for an unusual sella turcica in some patients with FXS. This report adds to the up to now sparse findings on sella morphology in FXS. Case Report: The young patient with genetically confirmed FXS was treated for a mandibular tumor. Cone beam tomography of the skull revealed a prominent sella turcica with flat tuberculum sellae and steep clivus. Conclusion: Unusual sella turcica formations can be observed in FXS. A correlation with other discreet changes of FXS-related skeletal development is likely.
\end{abstract}

Fragile X syndrome (FXS) is a relatively common syndrome associated with a variety of often subtle findings and symptoms (1). FXS is considered the most common Xchromosome-linked cause of mental retardation (2). Mental developmental disorders are frequently diagnosed in males. The disease also affects females (1). The cause of the disease is an alteration of the FMR1 gene coding for the fragilemental-retardation-1 protein (1). The cause is an expanding triplet-repeat in this gen. There is a correlation between the number of repeats and the severity of the disease. Numerous craniofacial anomalies have been described for FXS, such as a large head, long face, and large ears (2). However, in individual cases, the craniofacial findings may be very discretely developed or absent (1). One report detailed skeletal dysplasia of the skull base in FXS. It was described

This article is freely accessible online.

Correspondence to: Professor R. E. Friedrich, MD, DMD, Ph.D., FEBOMFS, Department of Oral and Craniomaxillofacial Surgery, Eppendorf University Hospital, University of Hamburg, Martinist. 52, D-2046 Hamburg, Germany. Tel: +49 40741053259, e-mail: rfriedrich@uke.de

Key Words: Fragile X syndrome, triple X syndrome, sella turcica, skull base, cone beam computed tomography. in detail that sella turcica development was considerably altered in FXS patients (3). Pathological sella morphology is also present in the majority of fetuses with known FXS (4). However, previous descriptions of sella morphology in FXS are based on only a few cases $(3,4)$. In the case presented, a radiological examination of the skull was performed in a FXS patient because of a jaw lesion, which revealed complementary findings to the sella region.

\section{Case Report}

The female patient, who was 16 years old at the time of the initial examination, has been treated at the oral and maxillofacial surgery clinic for a sensory disturbance of the right buccal skin, right mandibular swelling, and a subsequently diagnosed ipsilateral mandibular lesion. The mandibular lesion was fibrous dysplasia. Furthermore, the patient suffers from triple $\mathrm{X}$ syndrome. Diagnosis and treatment of the mandibular lesion already has been described in detail (5).

The skull base was also imaged on cone beam computed tomography (CBCT) of the facial skull (Viso G7, Planmeca, Helsinki, Finland) (Figure 1). On the sagittal cross-sectional images, the anterior wall of the sella is oblique and looks 'depressed' (3). The sella appears slightly egg-shaped. The posterior wall is thin, and its basis is small. Because the anterior wall slopes very obliquely dorsally and the base of the posterior sella originates on a very steeply sloping clivus, the posterior wall is relatively long (maximum length: $0.93 \mathrm{~cm}$ ). The sphenoid bone is pneumatized. The sphenoid cavity reaches the occipital surface of the clivus. Postsellar pneumatization of the sphenoid bone excludes the posterior wall of the sella. The spheno-occipital synchondrosis is no longer visible. A notch within the anterior or posterior wall is not observed. The upper part of the clivus surface has developed a small protuberance towards the brain side (Figure 1E).

In the sagittal plane, maximum horizontal diameter is $0.82 \mathrm{~cm}$, maximum length (tuberculum sellae to bottom of dorsal wall) is $1.32 \mathrm{~cm}$ and minimum tuberculum-posterior clinoid distance is $0.42 \mathrm{~cm}$. In coronal plane, maximum horizontal sella diameter is $0.88 \mathrm{~cm}$, maximum height of 


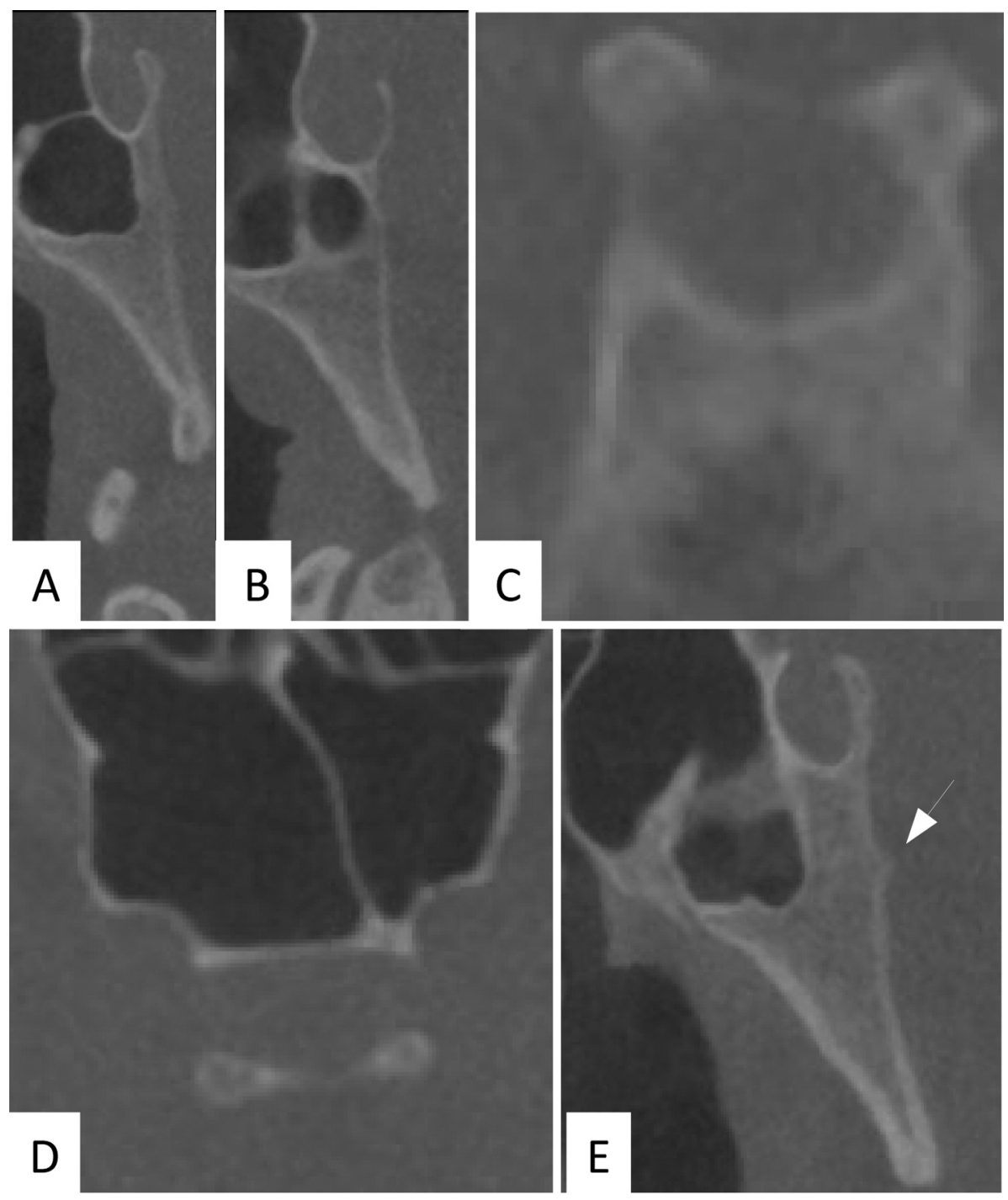

Figure 1. Sella turcica region in fragile X syndrome (FXS) on cone beam computed tomograms (CBCT), cropped images. (A) Prominent posterior clinoid process with relative narrow opening of the sella. (B) Very thin dorsum sellae, which is not pneumatized. (C) Round outline of the sella in coronal section at the level of the posterior wall. (D) Axial cross-sectional image of the sella region shows the very thin dorsum sellae. (E) Small protuberance of the clivus (arrow). The sectional image clearly shows the oblique course of the anterior wall of the sella turcica and the close positional relationship of the anterior and posterior clinoid process.

right posterior clinoid process is $1.07 \mathrm{~cm}$ (reference: horizontal line from deepest point of sella in coronal view), maximum height of left posterior clinoid process is $0.97 \mathrm{~cm}$ (reference: deepest point of sella in coronal view). NasionSella distance is $63.80 \mathrm{~mm}$. Sella - Basion distance is 42.66 $\mathrm{mm}$. Nasion-Sella-Basion (NSBa) angle is about $120^{\circ}$. Calculated sella volume is $830 \mathrm{~mm}^{3}$ (Figure 2) (Brainlab, Feldkirchen, Germany).

The radiological findings of the sella region certainly exclude further manifestation of the known fibrous dysplasia of the facial skeleton.

\section{Discussion}

The diagnostic significance of the sella turcica is primarily the bone's relationship to its essential functional content, the pituitary gland. Pathologies of the sella turcica are usually not early signs of disease of the pituitary gland (6). The interpretation of radiologic changes of this bone segment on plain radiographs for neurological diseases have become largely diagnostically irrelevant due to the superior sectional imaging techniques of magnetic resonance tomography and computed tomography in this field (6). Also, in standardized 


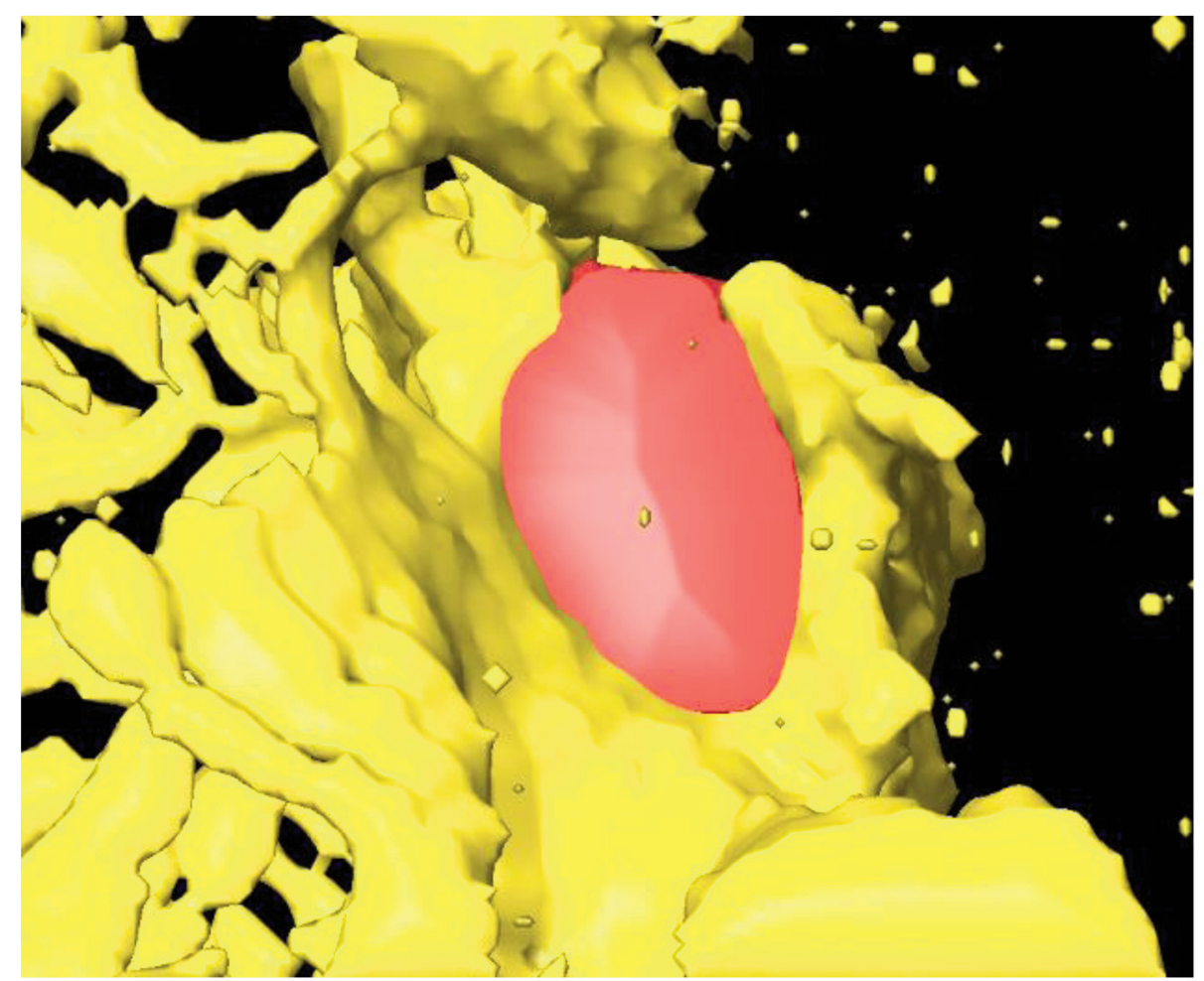

Figure 2. Three-dimensional reconstruction of sella turcica region derived from cone beam computed tomography of the skull base. Anterior is to the left. The cavum is marked three-dimensionally by the red surface. The oblique anterior wall and oval shape of the sella becomes clear in the $3 D$ reconstruction.

lateral cephalometry, even significant differences in the size and contour of the sella are often considered normal variations (6). However, the morphology of the sella turcica may offer clues to syndromic disease which can be assessed on plain radiographs $(7,8)$. Standardized cephalometric posterior-anterior or lateral projections are usually obtained for radiological survey of the skull in suspected syndromic skull malformations to make an initial assessment of the craniofacial skeleton $(6,7)$. On lateral cephalograms, sella turcica is a characteristic cup-shaped indentation in the center of the skull base facing the brain. On lateral cephalograms and $\mathrm{CBCT}$, the shape, size, inclination, and thickness of the walls as well as relationships to neighbouring skull base regions are assessed $(7,8)$. However, the shape and size of the sella are very variable (9-11). The type of sella in the sagittal section corresponds most closely to the oblique anterior sella according to Axelsson et al. (12).

Fragile X syndrome (FXS) and sella turcica. There is some information on the morphology of sella turcica at FXS. Kjaer et al. (3) examined 7 cases with FXS radiologically regarding skeletal dysplasia (age 2 years, 9 months to 21 years, 2 months, males: 6, female: 1). The sella was malformed in all cases where skull $\mathrm{x}$-rays were available for study $(n=5)$. The findings were individually variable. Oblique anterior wall of the sella was prominent in 2 cases, giving the impression of a cranially 'open' sella turcica (3). The dorsum sellae was extremely short developed in other two cases. One case had developed a sella bridge. The reproduction of radiological details in the figures of the patients represent only findings of males (3). In comparison to the reported five cases, the present case also shows an oblique, shallowly developed anterior sella wall merging into the sella bottom (Figure 1 and Figure 2). In contrast to previous reports $(3,4)$, the dorsum sellae is long, thin, and slightly curved. An 'open' sella (3) cannot be deduced from the radiographic findings. A steep inclination of the clivus was registered in 2 cases (3). This finding is also evident in the presented case. The volume of the sella is within the range of normal sella volume values published in recent studies (9-11).

Further studies on embryological specimen with known FXS revealed developmental defects of sella turcica (4). The authors examined the sella turcica in two embryos and recorded a pronounced notching of the anterior wall in one case and a very short dorsum sellae in the second case (4). Both findings were 
absent in the present case. The development of the dorsum sellae is controlled by the notochord $(7,8)$. Nasion-SellaBasion (NSBa) angle (inclination between anterior and posterior skull base) of the patient is below the expected mean value $\left(131.22^{\circ} \pm 4.73\right)(13)$.

Triple $X$ syndrome (TXS). In principle, the patient's known TXS could have had an influence on the skull base development. However, the facial findings of affected females are not very characteristic. A conspicuous radiological skull base has not been described so far in TXS. Reference bias to TXS may have an impact on the estimation of the frequency of syndrome-associated malformations (14). To our knowledge, cephalometric studies on TXS are not available. Isolated cases with facial co-morbidities potentially influencing bone development are probably sporadic (15). A case report with TXS and aplasia of a central incisor of the maxilla is interesting (16), because the fronto-maxillary developmental field is affected, whose central cranial endpoint is localized in the anterior wall of the sella $(7,8)$. Overall, an influence of the TXS on the sella morphology cannot be substantiated according to the current state of knowledge.

\section{Conclusion}

Sella turcica may have a conspicuous outline in some FXS cases. It is likely that many FXS cases remain undiagnosed due to low symptomatology. Unusual and described in a previous and the present report is the very oblique front wall of the sella. Assessment of sella turcica on standard skull radiographs may help classifying syndromes.

\section{Conflicts of Interest}

The Author declares that there are no conflicts of interest regarding this publication.

\section{Acknowledgements}

The assistance of Shih-Jan Chin, DMD, OMFS department, UKE, in making the three-dimensional reconstructions of the skull base is gratefully acknowledged. The Author would like to thank the patient and her parents for permission to publish the case report.

\section{References}

1 Lozano R, Rosero CA and Hagerman RJ: Fragile X spectrum disorders. Intractable Rare Dis Res 3(4): 134-146, 2014. PMID: 25606363. DOI: $10.5582 /$ irdr.2014.01022

2 Stone WL, Basit H and Los E: Fragile X Syndrome. StatPearls [Internet], 2021. PMID: 29083768.

3 Kjaer I, Hjalgrim H and Russell BG: Cranial and hand skeleton in fragile X syndrome. Am J Med Genet 100(2): 156-161, 2001. PMID: 11298378. DOI: 10.1002/ajmg.1226
4 Hjalgrim H, Fisher Hansen B, Brondum-Nielsen K, Nolting D and Kjaer I: Aspects of skeletal development in fragile $\mathrm{X}$ syndrome fetuses. Am J Med Genet 95(2): 123-129, 2000. PMID: 11078561. DOI: 10.1002/1096-8628(20001113)95:2<123::aid-ajmg6>3.0.co;2u

5 Friedrich RE, Kohlrusch FK and Luebke AM: Symptomatic mandibular fibrous dysplasia with concurrent triple $\mathrm{X}$ - and premutation stage fragile-X-syndrome: Case report with short literature survey. Anticancer Res 39(12): 6769-6780, 2019. PMID: 31810942. DOI: 10.21873/anticanres.13892

6 Bonneville JF and Dietemann JL: Radiology of the sella turcica. Springer-Verlag, Berlin -Heidelberg - New York, pp. 41-42, 1981.

7 Kjaer I: Etiology-based dental and craniofacial diagnostics. Wiley \& Sons, Hoboken, 2016.

8 Kjær I: Sella turcica morphology and the pituitary gland-a new contribution to craniofacial diagnostics based on histology and neuroradiology. Eur J Orthod 37(1): 28-36, 2015. PMID: 23159420. DOI: $10.1093 /$ ejo/cjs091

9 Kucia A, Jankowski T, Siewniak M, Janiszewska-Olszowska J, Grocholewicz K, Szych Z and Wilk G: Sella turcica anomalies on lateral cephalometric radiographs of Polish children. Dentomaxillofac Radiol 43(8): 20140165, 2014. PMID: 25182120. DOI: $10.1259 / \mathrm{dmfr} .20140165$

10 Taner L, Deniz Uzuner F, Demirel O and Güngor K: Volumetric and three-dimensional examination of sella turcica by conebeam computed tomography: reference data for guidance to pathologic pituitary morphology. Folia Morphol (Warsz) 78(3): 517-523, 2019. PMID: 30444524. DOI: 10.5603/FM.a2018.0106

11 Al-Mohana RAAM, Muhammed FK, Li X and Lubamba GP: The bridging and normal dimensions of sella turcica in Yemeni individuals. Oral Radiol: 1-9, 2021. PMID: 34143355. DOI: 10.1007/s11282-021-00541-7

12 Axelsson S, Storhaug K and Kjaer I: Post-natal size and morphology of the sella turcica. Longitudinal cephalometric standards for Norwegians between 6 and 21 years of age. Eur J Orthod 26(6): 597-604, 2004. PMID: 15650069. DOI: 10.1093/ejo/26.6.597

13 Segner D and Hasund A: Individualisierte Kephalometrie. HansaDont Verlag, Hamburg, 2003.

14 Otter M, Schrander-Stumpel CT and Curfs LM: Triple X syndrome: a review of the literature. Eur J Hum Genet 18(3): 265-271, 2010. PMID: 19568271. DOI: 10.1038/ejhg.2009.109

15 Ferrazzo KL, Payeras MR, Ferrazzo VA and Mezomo MB: Craniofacial and dental manifestations of triple $\mathrm{X}$ syndrome associated with congenital hypothyroidism: a case report. Spec Care Dentist 34(3): 156-159, 2014. PMID: 24117978. DOI: $10.1111 / \mathrm{scd} .12050$

16 Miura M, Kato N, Kojima H and Oguchi H: Triple-X syndrome accompanied by single maxillary central incisor: case report. Pediatr Dent 15(3): 214-217, 1993. PMID: 8378162.

Received August 20, 2021

Revised September 2, 2021

Accepted September 3, 2021 